
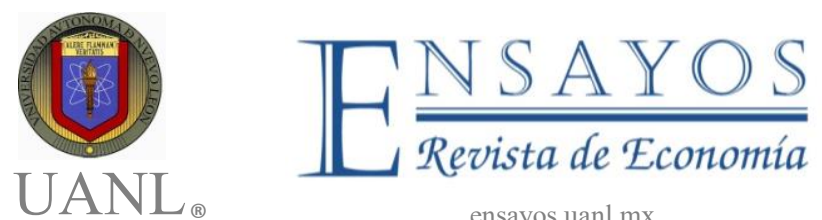

ensayos.uanl.mx

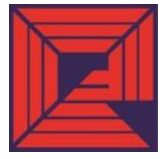

Facultad de Economía

\title{
Interrelación entre la competitividad internacional y el desarrollo humano en la región Asia-Pacífico
}

\section{Interrelation Between International Competitiveness and Human Development in the Asia-Pacific Region}

\section{América Ivonne Zamora Torres* Mitchell Rigel Ortiz Zamora*}

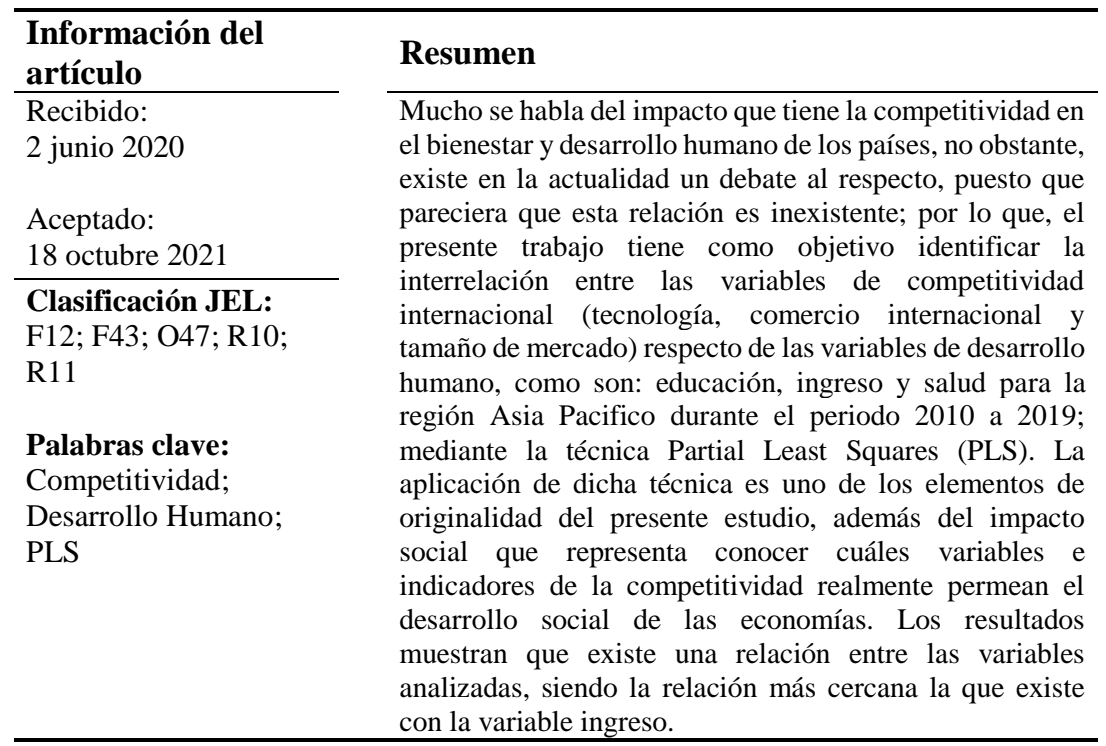

*Universidad Micoacana de San Nicolas de Hidalgo, americazt@ hotmail.com; ${ }^{* *}$ Instituto Tecnológico de Morelia

ISSN Electrónico: 2448-8402 | ISSN Impreso: 1870-221X | @2021 Los autores @ @

Citar así: Zamora Torres, A.I. y Ortiz Zamora, M.R. (2021). Interrelación entre la competitividad internacional y el desarrollo humano en la región Asia-Pacífico. Ensayos Revista de Economía, 40(2), 189-214, DOl:10.29105/ensayos40.2-4 


\begin{tabular}{|c|c|}
\hline Article information & Abstract \\
\hline $\begin{array}{l}\text { Received: } \\
2 \text { June } 2020\end{array}$ & $\begin{array}{l}\text { A lot has been said about the impact of competitiveness on } \\
\text { both the human development and wellbeing of the } \\
\text { population of a country. Yet, this is a contested subject due }\end{array}$ \\
\hline $\begin{array}{l}\text { Accepted: } \\
18 \text { October } 2021\end{array}$ & $\begin{array}{l}\text { to the almost non-existent nature of this relationship. This } \\
\text { paper aims to identify the interrelation between the }\end{array}$ \\
\hline $\begin{array}{l}\text { JEL Classification: } \\
\text { F12; F43; O47; R10; } \\
\text { R11 }\end{array}$ & $\begin{array}{l}\text { market size, and international trade) and those regarding } \\
\text { human development like education, income, and health, } \\
\text { for the Asia-Pacific region in the period from } 2010 \text { to } \\
\text { 2019; by using the Partial Least Squares (PLS) technique. }\end{array}$ \\
\hline $\begin{array}{l}\text { Keywords: } \\
\text { Competitiveness; } \\
\text { Human Development; } \\
\text { PLS }\end{array}$ & $\begin{array}{l}\text { The application of this technique is one of the original } \\
\text { elements of the present study and the social impact } \\
\text { represented by knowing which variables and indicators of } \\
\text { competitiveness permeate the social development of } \\
\text { economies. The results show a relationship between the } \\
\text { variables analyzed, being the closest relationship with the } \\
\text { variable income. }\end{array}$ \\
\hline
\end{tabular}

\section{Introducción}

Un gobierno soberano posee una extensa cantidad de objetivos a cumplir, uno de los cuales debe ser proveer a sus ciudadanos con una mejora en su calidad de vida (Costanza et al., 2008), qué tan efectivos son los gobiernos alcanzando dicha meta es debatible, y su eficacia varía de acuerdo con la época, las políticas públicas y las áreas de desarrollo en las que el gobierno decida enfocarse; una de las cuales es, por supuesto, la competitividad internacional del país. No obstante, es sencillo caer en la suposición de que, si las economías son más competitivas en un nivel internacional, esto se traduce necesariamente en un mayor nivel de bienestar para la población (Buendía, 2013) (Lombana y Rozas, 2009).

A inicios del siglo pasado, con la constante y creciente presencia de la globalización, no solo en el mundo sino en los ámbitos macroeconómicos y de comercio internacional, cada vez se volvió más evidente para las naciones del mundo, que mejorar su competitividad en términos de otros países es un requisito para progresar como economía (Calva, 2009). Así mismo, con el tiempo, al incrementar y trabajar en el desarrollo de los indicadores de competitividad internacional, se concibió el pensamiento de que el crecimiento en estos indicadores causaba un impacto, fuese substancial o inapreciable, sobre el desarrollo humano de una sociedad. Es innegable el hecho de que existe cierta coherencia lógica en la explicación de porqué este sería el caso, después de todo, el mejoramiento de un aspecto de la sociedad, en este caso la economía internacional, debe, por lo tanto, conducir al mejoramiento de otros aspectos en la misma sociedad. 
La teoría económica convencional sostiene que existe una correlación directa entre la competitividad y el nivel de vida de los habitantes de un país, por lo que resulta primordial para todo gobierno mantener altas tasas de crecimiento en la economía a lo largo del tiempo (Jiménez, 2011). Pequeñas variaciones porcentuales en el crecimiento del Producto Interno Bruto, durante largos periodos de tiempo, terminan generando grandes diferencias en términos de riqueza total. Como evidencia empírica de la afirmación anterior, encontramos el trabajo de Barro y Sala i Martín (2012), en el que se afirma que si la economía de los Estados Unidos hubiera crecido en promedio 1\% menos, durante el periódo 1870-2000, para ese último año, sus niveles de PIB per cápita hubiesen sido similares a los de México o Polonia.

Sin embargo, a pesar de que se ha trabajado bajo esta suposición, han existido ciertos casos que desafían este paradigma, especialmente el caso de Chile en 2000, donde diversos autores como Miquel (2015a), Miquel (2015b), OCDE(2015) y Mussi y Afonso (2008), entre otros, observan el fenómeno de cómo este país, a pesar de contar con indicadores de competitividad positivos, sanos, en amplia forma y creciendo, comenzó a experimentar una significativa disminución en los índices relacionados con el desarrollo humano y la calidad de vida de su población, hecho que va en contra de la suposición inicial.

Hoy en día, el peso económico de la Región Asia Pacifíco es muy significativo, puesto que para 2018, solamente las economías de APEC (Foro de Cooperación Económica Asia Pacífico), es decir, sus veintiún miembros, representaban el $56.75 \%$ del Producto Interno Bruto Mundial y el $47 \%$ del comercio mundial. El peso económico de los países que conforman dicha región no se expresa solo en términos absolutos, puesto que la región se caracteriza por un dinamismo mayor al promedio mundial (APEC, 2021).

Se observa que en el periódo 2000-2018, la República Popular China ha tenido un crecimiento del PIB per cápita promedio de $8.53 \%$, le siguen Viet Nam con crecimiento promedio anual del 5.39\%, Indonesia (3.9\%), Rusia (3.8), Perú (3.79\%), Filipinas (3.51\%), Corea del Sur (3.49), Tailandia del $3.46 \%$ y Singapur, del 3.35\% (APEC, 2021). Dichos resultados contrastan con los resultados del PIB per cápita de la mayoría de las naciones que no son del Sureste Asiatico, como Canada, Nueva Zelanda, Australia, Estados Unidos o México cuyas tasas de crecimiento anual del PIB per cápita son muy inferiores al $2 \%$ anual.

Derivado de lo anterior, el objetivo de este estudio es identificar la interrelación entre las variables de competitividad internacional (tecnología, comercio internacional y tamaño de mercado) respecto de las variables de desarrollo humano, como son: educación, ingreso y educación para la región Asia Pacifico (siendo esta la región más dinámica comercialmente hablando), 
durante el periodo 2010 a 2019; es decir, se pretende analizar si los factores que crean riqueza (competitividad) también generan bienestar para la población (desarrollo humano) en los países analizados. Cabe señalar que el periodo de análisis obedece a las restricciones de disponibilidad de datos. Partiendo del supuesto teórico que asegura existe una relación de impacto positivo de las variables de competitividad internacional (tecnología, comercio internacional y tamaño de mercado), en las variables de desarrollo humano (educación, ingreso y educación), para la región Asia Pacifico, durante el periodo 2010 a 2019.

Esto permitirá definir si, cuando un país aumenta sus índices de competitividad internacional, necesariamente causará un crecimiento en los índices de desarrollo humano. Para ello se utiliza, como herramienta metodológica, la técnica de Partial Least Squares (PLS), la cual permite determinar el impacto y la magnitud de las interrelaciones entre una o más variables, pudiendo traducir así la competitividad internacional en calidad de vida. Para dicho análisis, se utilizó una serie de ocho variables de competitividad internacional con ocho índices de desarrollo humano, a través de nueve años (2010 a 2019), para once países (Australia, Canadá, Chile, China, Corea del Sur, Estados Unidos, Japón, México, Nueva Zelanda, Rusia y Singapur) de la región AsiaPacífico.

El presente documento consta de cinco secciones: en la primera, se encuentra la revisión literaria con respecto a otros estudios, los cuales muestran el impacto de los indicadores de competitividad sobre el desarrollo humano, ya sea a menor o mayor escala o en otras regiones; posteriormente, en el segundo apartado, se encuentra la aproximación metodológica seguida de las variables latentes y observadas; en el tercer apartado, se presenta la selección de variables; en la cuarta sección, se analizan los resultados obtenidos para, finalmente, en el quinto apartado mostrar las principales conclusiones y la discusión al respecto.

\section{Marco teórico y revisión de literatura}

Cuando un país o una empresa participan en los mercados internacionales, es lógico exigir, que sea competitivo, sin embargo: ¿qué es ser competitivo? La competitividad es considerada un tema fundamental en todos los sectores económicos, tanto en el nivel nacional, regional, industrial y empresarial. En el ámbito internacional, dentro de un proceso de globalización, se exige a las empresas ser competentes, eficaces y sustentables en el manejo de los recursos financieros, humanos, naturales y tecnológicos, entre otros, para poder enfrentar los retos que representan los mercados tanto domésticos o nacionales, como los internacionales. 
El concepto de competitividad es extenso, ha sido tratado e interpretado por diferentes investigadores, académicos e intelectuales, así como por organismos e instituciones desde finales de la década de 1970. Al mismo tiempo, la competitividad es justificante para determinar la administración de una empresa, que produzca beneficios y bienestar a la comunidad, o bien, para conocer el nivel de competitividad entre los países, así como, para decretar políticas públicas por parte de los gobiernos e impulsar el desarrollo de cierto sector o industria. Por otro lado, existe una gama de variables e indicadores para medirla, así como una cantidad de factores que parecen tener un impacto sobre el mismo concepto (Krugman 1994, 1996; Porter, 1990; Abdel y Romo, 2004). Como se mencionó, anteriormente, frecuentemente se abordan temas de competitividad en términos internacionales, asumiendo que estos poseen un impacto significativo sobre el desarrollo humano de una sociedad. No obstante, la magnitud y presencia de esta conexión ha tratado de ser estudiada y comprendida, al menos mediante el uso de diferentes técnicas, variables y con el uso de sujetos de estudio provenientes de regiones diversas, distintos a los de la presente investigación.

En la formulación de políticas públicas, en las distintas áreas económicas, en los organismos mundiales, así como en la academia, el constructo "competitividad" es, sin duda alguna, uno de los menos comprendidos y más ambiguos; esto se debe a los distintos enfoques que continuamente se hacen para definirlo y analizarlo, y sobre todo a los distintos niveles en los que se aplica (país, región, industria y empresa).

Porter (1990) defiende la competitividad tanto en nivel macro, como microeconómico; el grado en que un país, estado, región o empresa produce bienes o servicios bajo condiciones de libre mercado, enfrentando la competencia de los mercados nacionales o internacionales, mejorando simultáneamente los ingresos reales de sus empleados y consecuentemente la productividad de sus empresas. Porter plantea que las empresas con ventajas competitivas transforman a las ciudades, territorios y regiones en competidoras $\mathrm{y}$, por lo tanto, las naciones poseedoras de esa cualidad, más la suma de sus empresas competitivas y generadoras de rentabilidad, dan como resultado países con abundante riqueza para sus habitantes y mayor bienestar general.

La literatura al parecer confunde la "competitividad" con el rendimiento superior de las empresas, y últimamente, se la ha dado más difusión en la gestión estratégica. Palabras como "alto nivel de vida", "ingreso nacional per cápita", están asociadas a un país, y también "productividad", "beneficio" y "valor", vinculados a la competitividad de las empresas (Bosse, Philips y Harrison, 2009). 
Otras propuestas sobre el significado de competitividad, basado en la productividad, son las planteadas por Krugman $(1990,1994)$ y Porter (1990), quienes establecen el criterio para los fundamentos de la perspectiva de la competitividad, actualizada en la literatura económica y de gestión.

La internacionalización de las economías fomenta en los países y las empresas que impulsen el incremento de la competitividad en los mercados globales. Sin embargo, no se subraya la importancia de la productividad como un factor relevante de la competitividad, es decir, establecer a la productividad, es crear ventaja competitiva, por esta razón, existe una cercana y directa relación entre los dos conceptos (Buckley, Pass y Prescott, 1998).

La competitividad posibilita a los países y a las empresas a enfrentar positivamente la competencia internacional, ya sea en el ámbito nacional o doméstico, como en el mundial. Por otro lado, la productividad especifica la relación entre los recursos disponibles, las unidades que se producen y su valor; en consecuencia, el mejoramiento de esta permite potenciar la capacidad competitiva. En el entorno económico, el crecimiento está asociado con diversos aspectos y uno de ellos es la competitividad, se debe empezar a proponer como objetivo prioritario la importancia de la productividad, toda vez que su estímulo refleja el uso eficiente o no de los recursos con que cuentan los países, las industrias, los sectores y las empresas (Wysokinska, 2003).

Como se mencionó anteriormente, la competitividad se puede calificar en diferentes niveles de análisis: país y/o región, industria y/o sector y empresa. La competitividad del país incluye dimensiones macroeconómicas (PIB, estabilidad, acceso a los mercados, complejidad en la regulación, entre otras). La competitividad industrial hace referencia a la infraestructura regional; y, la competitividad empresarial hace una revisión interna de la organización. La competitividad de la firma está supeditada a la relación con la competitividad en nivel país y sector, así como con su propio nivel (Buckley, Pass y Prescott, 1998; Wysokinska, 2003).

Siudek y Zawojska (2013), hacen énfasis en la competitividad en el nivel firma, ya que señalan que una empresa competitiva es aquella que tiene la capacidad de generar ganancias con una participación de mercado significativa. No obstante, para ser considerada competitiva, la empresa debe utilizar sus recursos, su capacidad para producir los productos y servicios adecuados, con calidad, un precio aceptable y en la cantidad correcta, así como comercializar, de manera efectiva, sus productos al mercado consumidor. En el largo plazo, en un sistema de libre comercio, la competitividad se mide por la capacidad de la empresa para continuar con el negocio y proteger sus inversiones, generando rentabilidad sobre las inversiones, pero también garantizar empleo a futuro. 
Se debe entender cabalmente a la competitividad, por lo que es fundamental determinar sus características endógenas y exógenas, una de ellas es la productividad, al incrementarse, produce el desarrollo tecnológico, la reducción de costos, así como con una mejora de la competitividad. Finalmente, la competitividad de un país, industria y empresa está determinada por la productividad, si bien es un factor clave, también convergen otros elementos, como es el caso de la rentabilidad, la posición competitiva, la participación del mercado interno y externo, así como sus relaciones interempresariales, entre otros (Boone, 2008).

Dixit y Stiglitz (1977) y Krugman (1979b y 1980), investigan sobre las teorías contemporáneas de la competitividad, modelan la competencia imperfecta con productos altamente diferenciados y curvas de demanda con pendiente negativa.

Por su parte, Krugman (1991), dentro del mercado competitivo imperfecto, afirma que los rendimientos crecientes a escala, es decir, la productividad, determina las ventajas comerciales y la dirección de las exportaciones, en estudios posteriores, relaciona la competitividad con la producción (Krugman, 1991).

Asimismo, Porter (1990), en la literatura sobre gestión estratégica, desarrolla las teorías de la competitividad internacional basadas en su modelo "Diamante", el cual explica los cuatro determinantes de la competitividad específicos para cada país, región, industria y empresa: 1) condiciones de los factores de producción; 2) condiciones de la demanda interna; 3) industrias relacionadas o sectores conexos y de apoyo (clústeres); 4) estrategia, estructura y rivalidad empresarial, con dos factores externos: a) el gobierno que favorece las condiciones para el suministro de los factores de producción claves; y b) eventos fortuitos que ocurren fuera del control de las empresas y generan interrupciones que influyen en la ganancia o pérdida de la posición competitiva.

Prahalad y Hamel (1990), presentan la competitividad de la empresa a corto plazo, de acuerdo con la visión basada en recursos, donde el resultado se da por los atributos de precio y rendimiento de los productos existentes; en el largo plazo, deriva de la capacidad de la empresa para fabricar productos a menor costo y más rápido que la competencia.

Buckley, Pass y Prescott (1998), configuran un modelo de competencia en tres dimensiones: 1) desempeño competitivo; 2) potencial competitivo (recursos utilizados para generar desempeño); y 3) proceso competitivo (gestión estratégica de la empresa). 
El comportamiento de la capacidad productiva acelera o retrasa a una economía y es una variable fundamental; por tanto, contextualizarla, medirla y evaluarla es de primordial importancia para formular políticas que estimulen su desarrollo. Prescott (1998), hace énfasis en la importancia de la productividad como fuente de crecimiento, y destaca que su estudio es insuficiente. Por otro lado, el comportamiento de esta variable explica aproximadamente el $40 \%$ del crecimiento económico en los países en vías de desarrollo y, el 80\%, en las naciones desarrolladas (Reyes, 2010).

De acuerdo con Porter $(1991,1997)$ y Krugman (1996), la productividad es el elemento fundamental de la competitividad de un país, la competitividad no se hereda sino se crea y está en función, como primer elemento, de la capacidad de su sector industrial para adoptar innovaciones tecnológicas, que se traducen en un incremento en la productividad. Por otro lado, cuando se rompen las barreras del comercio internacional, la localización de las actividades económicas tenderá a concentrarse en el territorio central, debido a consideraciones vinculadas con ventajas absolutas.

Existen diversos indicadores de la competitividad de un país como medida relativa que compara el desempeño económico de alguna unidad de análisis, frente al resto que forman el universo de estudio, los cuales son: 1) el comportamiento de las exportaciones; 2) el balance en la cuenta corriente; 3) la tasa de cambio real basada en los índices de precios al consumidor; 4) el costo unitario de la mano de obra; 5) los precios de bienes y servicios para el consumo interno; y 6) la participación de los sueldos y salarios en el valor agregado (Marsh y Tokarik, 1994; Malecki, 1997).

De acuerdo con el Instituto Nacional de Estadística, Geografía e Informática (INEGI), la competitividad de una economía es el resultado de una compleja correlación de diferentes indicadores: los costos de los insumos intermedios y de los factores de producción, la productividad de los mismos, los precios de los productos terminados, el régimen impositivo, la organización de los mercados y los sistemas de distribución, entre muchos más; por esta razón, es difícil expresar la competitividad en forma de un solo indicador. Por lo tanto, el INEGI presenta una serie de indicadores que influyen de manera significativa en la posición competitiva de nuestro país. Así, para la economía mexicana, se utilizan los siguientes indicadores de competitividad: 1) tasas de interés; 2) costos unitarios de la mano de obra; 3) productividad parcial del trabajo; 4) promedios arancelarios entre México y EE. UU.; 5) participación en la importación manufacturera de EE. UU.; y 6) penetración de las exportaciones mexicanas en los mercados de EE. UU., y la Unión Europea (INEGI, 1995). 
Los indicadores de competitividad no son variables explicativas, únicamente representan medidas cuantitativas del éxito competitivo de una unidad de análisis. Las variables explicativas hacen referencia a las ventajas competitivas que serán los factores o determinantes de la competitividad. En materia macroeconómica, estos factores se especifican hacia la promoción de la apertura comercial, estímulos al desarrollo tecnológico, desregulación económica y política industrial (Noyola, 1991).

En cuanto a las mediciones de competitividad, existen diferentes aproximaciones. Por ejemplo, "La Medición de la Competitividad en México," los investigadores Garduño, Ibarra y Dávila (2013) llevan a cabo una crítica objetiva con respecto a los distintos indicadores de competitividad que han sido utilizados, en México, con el propósito de obtener una mejor y más clara interpretación de los datos provistos por cada uno de ellos. Los indicadores que este estudio analiza son: el Índice Global de Competitividad, diseñado por la Escuela de Graduados en Administración Pública y Política Pública (EGAP), el Índice de Competitividad Urbana del Instituto Mexicano para la Competitividad (IMCO), el Índice de Competitividad de las Ciudades Mexicanas, el Índice de Competitividad Revelada (ICR) y el Índice de Competitividad Sistémica desarrollada por Aregional. Los investigadores concluyen que existe un gran número de contradicciones entre los resultados provistos por los índices, particularmente, los de las regiones de menor escala geográfica.

Ordóñez (2011), en su publicación de: “¿Competitividad para qué? Análisis de la relación entre Competitividad y Desarrollo Humano en México", revela que el actuar del gobierno es de fundamental importancia para propiciar el desarrollo de una población, especialmente en adecuado manejo de las políticas públicas que este implemente. Además, enfatiza que el Estado es uno de los factores con mayor influencia en un territorio, para generar las condiciones tanto de competitividad como de desarrollo humano, en sus ciudadanos. El estudio de Ordóñez hace uso de cuatro indicadores distintos bajo periodos de tiempo similares: la captación banca comercial per cápita en un periodo 2003-2007, el PIB/PEA en los años 2003-2008, el IDH sin componente de ingreso entre 2000-2004 y, finalmente, la Inversión Extranjera Directa per cápita en el periodo 1994-2009, encontrando que las entidades federativas más competitivas son, a su vez, aquellas que presentan un mayor grado de desarrollo humano.

Otro estudio analiza una serie de indicadores e índices, así como su valor subjetivo para cuantificar el bienestar y la cohesión social en una población dada, tal es el caso de la región del País Vasco. Entre los indicadores que analiza, se encuentran: las ventajas y desventajas de hacer uso del PIB, el Índice de Desarrollo Humano, el Î́ndice de Progreso Social, el Índice 
Canadiense de Bienestar, así como cada uno de los elementos que integran a dichos indicadores, tales como la expectativa de vida, el radio de alfabetismo y escolarización, vitalidad de las comunidades, salud subjetiva de la población, entre otros. Esto concluye con la sugerencia de una nueva aproximación a la competitividad, que considere dimensiones más complejas, desde aspectos personales del individuo (Larrea, 2018).

En el libro "Competitividad, Crecimiento y Capitalización de las Regiones Españolas" se vincula la idea de la competitividad a un valor positivo que se halla estrechamente relacionado con un comportamiento económico eficiente en España, lo que lleva a que se note, principalmente, que las diferencias absolutas de productividad adquieren una relevancia en el comercio interregional que no posee en ámbitos entre estados. Es decir, los indicadores poseen un valor mayor en cuestiones geográficamente amplias. Los indicadores que el trabajo analiza, son aquellos relacionados con algún aspecto de la descomposición del PIB per cápita, tales como la productividad del trabajo, la tasa de empleo y la proporción de la población en edad de trabajar sobre población total, así como indicadores basados en agregación de un conjunto de variables representativas, estos son la medición de recursos humanos disponibles, la innovación tecnológica, la infraestructura relacionada con la accesibilidad y el entorno productivo. Se concluye que el segundo conjunto de indicadores es mejor para estimar tasas reales de bienestar subjetivo, ya que no mide la competitividad en sí sino sus causales (Mas et al., 2007).

Como se observa, numerosos investigadores han propuesto diversas formas de medir la calidad de vida de los individuos desde diferentes aspectos, los cuales se han puesto en práctica por distintas organizaciones. El Programa de las Naciones Unidas para el Desarrollo hace uso del Índice de Desarrollo Humano en su informe anual Reporte del Desarrollo Humano 2019, el cual obtiene al calcular distintos índices de educación, salud, expectativas de vida y estándares de vida. En la educación, se hace uso de indicadores tales como la relación nacional entre el número de profesores y el número de estudiantes, los resultados del Programa Internacional para la Evaluación de Estudiantes (PISA por sus siglas en inglés) y del porcentaje de la población con educación de nivel secundaria. Así mismo, para medir el bienestar en términos de salud, se cuenta el número de camas y médicos por cada 10,000 habitantes así como la esperanza de vida, de cada país (PNUD, 2019).

Por su parte, la Red de Soluciones para el Desarrollo Sostenible, con el propósito de crear un indicador que tome en cuenta a la felicidad como medida de calidad de vida, utiliza lo que es conocido como el Índice Global de la Felicidad, el cual hace uso de indicadores subjetivos (tales como: la generosidad, la libertad percibida para tomar decisiones, etc.) que se obtienen 
mediante las encuestas directas y presenciales aplicadas a la población evaluada, así como indicadores cuantificables, entre ellos el PIB per cápita, la esperanza de vida en años de la población, índices de corrupción, satisfacción con el gobierno, seguridad percibida, entre otros (Helliwell, Layard, Sachs \& De Neve, 2020).

Lonska y Boronenko (2015), en su trabajo Ranking Competitiveness and Human Development in Global Comparative Researches, pretenden comprobar la existencia de una relación bidireccional entre los análisis de desarrollo humano en los países con su contraparte de crecimiento económico, tomando como base las comparativas globales del Reporte de Desarrollo Humano del Programa de Desarrollo de la Organización de las Naciones Unidas y el Reporte de Competitividad Global del Foro Económico Mundial. Para llevar a cabo el estudio, se utilizaron los doce pilares que integran el índice de desarrollo mundial junto con aquellos que componen el índice de desarrollo humano. Al final, lograron determinar que existe una correlación estadísticamente significativa para todas las variables que fueron aplicadas, demostrando que un más rápido crecimiento en la competitividad, se traduce en un incremento en la velocidad del desarrollo humano dentro de un país.

Adicionalmente, en el estudio sobre del Índice de la Calidad de Vida Física, se busca obtener formas de medir el bienestar de la población, sin tomar en cuenta indicadores macroeconómicos como el PIB; para calcularlo, se usaron medidas con un impacto directo en la población, razón por la cual se utilizaron la tasa de mortalidad infantil, la esperanza de vida en años de la población y la tasa de alfabetismo en adultos, como indicadores (Morris, 1979).

Estos estudios demuestran la amplia contingencia en cuanto al número, selección y peso que deben tener ciertos indicadores sobre otros, para efectivamente llevar a cabo comparativas de competitividad. No obstante, dicho tema pierde esclarecimiento al pretender identificar cuáles índices de competitividad en un entorno macroeconómico son los que poseen un impacto, efecto o relación profunda con aquellos indicadores de bienestar subjetivo, así como de desarrollo humano en su población.

\section{Aproximación metodológica: Mínimos cuadrados parciales (Partial Least Squares, PLS)}

Los modelos de ecuaciones estructurales (MEE) se han convertido en uno de los desarrollos recientes e importantes del análisis multivariante y su uso se ha extendido entre las ciencias sociales (Fornell, 1982), por ello, es posible utilizar modelos cada vez más complejos, con el surgimiento de técnicas como la Modelación de Ecuaciones Estructurales (Structural Equation Modeling, SEM), que permite realizar regresiones múltiples entre variables latentes 
(Batista Foguet \& Coenders Gallart, 2000). Claes Fornell (1982) denomina a estos modelos de ecuaciones estructurales, análisis multivariante de segunda generación.

La característica común de las técnicas que se acogen bajo esta etiqueta es el reconocimiento metodológico de que la teoría científica implica tanto variables empíricas como abstractas. En este sentido, el propósito de los análisis multivariante de segunda generación es ayudar a vincular datos y teoría. Fornell (1982) subraya que las metodologías de análisis multivariante de segunda generación enfatizan los aspectos acumulativos del desarrollo de la teoría, por lo cual, el conocimiento a priori es incorporado dentro del análisis empírico. Este conocimiento a priori puede originarse a partir de la teoría, de descubrimientos empíricos previos o del diseño de la investigación. Debido a que estos métodos pueden combinar y confrontar la teoría con datos empíricos, ofrecen el potencial para una explicación científica, que vaya más allá de la asociación o descripción empírica.

En este sentido, los MEE son técnicas multivariantes que combinan aspectos de la regresión múltiple (examinando relaciones de dependencia) y análisis factorial (que representan conceptos inmedibles -factores- con variables múltiples), para estimar una serie de relaciones de dependencia interrelacionadas simultáneamente (Cepeda y Roldán, 2004).

De los resultados obtenidos, es posible construir un modelo que permita observar las interrelaciones entre las variables. En este caso, se utiliza la técnica PLS, una técnica de Modelación de Ecuaciones Estructurales, el cual se enfoca en maximizar la varianza de las variables dependientes explicadas por las independientes (Loehlin, 1998). A través del presente modelo, se busca contribuir al entendimiento de las interrelaciones entre variables que determinan el desempeño competitivo de una compañía; y este conocimiento podría afectar directamente el desempeño del negocio (Johnson, 1997).

De tal forma que, el objetivo perseguido por la modelización PLS es la predicción de las variables dependientes (tanto latentes como manifiestas). Esta meta se traduce en un intento por maximizar la varianza explicada (R2) de las variables dependientes, lo que lleva a entender que las estimaciones de los parámetros estén basadas en la capacidad de minimizar las varianzas residuales de las variables endógenas.

Chin (1998) establece tres distinciones básicas para elegir entre MBC y PLS:

1. Que los constructos subyacentes sean modelados como indeterminados o determinados. 
2. El alto o bajo grado de confianza que el investigador tenga en el modelo teórico y en la teoría auxiliar que vincula las medidas (variables observables) con los constructos.

3. Que el investigador esté orientado hacia el cálculo de los parámetros o hacia la predicción.

Si las respuestas del investigador se inclinan hacia las segundas opciones, entonces PLS se convierte en el enfoque más adecuado (Cepeda y Roldán, 2004).

PLS tiene la capacidad de tratar con modelos muy complejos con un gran número de constructos e interrelaciones, permite trabajar con muestras relativamente pequeñas, hace suposiciones menos estrictas sobre la distribución de datos, pudiendo trabajar con datos nominales, ordinales o de intervalo, tal es el caso de la presente investigación. Además, se ha demostrado que los métodos matemáticos de PLS son bastante rigurosos y robustos. PLS puede ser una herramienta poderosa por las mínimas demandas de escalas de medición, tamaño de muestra y distribuciones residuales (Ortega y Bonales, 2014).

La idea básica del PLS es la siguiente: primero, los pesos de las relaciones que vinculan los indicadores a sus respectivas variables latentes, son estimados; segundo, se calculan los case values para cada variable latente basado en un promedio ponderado de sus indicadores. Finalmente, estos case values son usados en un grupo de ecuaciones de regresión para determinar los parámetros de los coeficientes paths o estructurales. El algoritmo regresa nuevamente a las relaciones del modelo de medición, en donde nuevos pesos son calculados, y el proceso continuo iterativamente hasta que se alcanza la convergencia de los pesos.

El algoritmo PLS, genera cargas entre los constructos reflexivos y sus indicadores y pesos entre los constructos formativos y sus indicadores. Este algoritmo también produce coeficientes de regresión estandarizados entre constructos, y coeficientes de determinación múltiple para todos los constructos endógenos del modelo.

En un modelo PLS, la confiabilidad individual del ítem es valorada, examinando las cargas o correlaciones simples de los indicadores con su respectivo constructo. Las variables latentes con indicadores formativos deben ser evaluadas en función de sus pesos y no de sus cargas. Para examinar qué tan bien los indicadores son explicados por su variable latente se debe usar un índice llamado comunalidad, este mide cuánta de la varianza de una cierta variable manifiesta se le puede adjudicar a su variable latente (Haenlein y Kaplan, 2004). Así pues, se ha demostrado que los métodos matemáticos de PLS son bastante rigurosos y robustos (Haenlein \& Kaplan, 2004). En 
resumen, el PLS puede ser una herramienta poderosa por las mínimas demandas de escalas de medición, tamaño de muestra y distribuciones residuales (Chin, 2010).

Cabe señalar que el PLS es una técnica comúnmente empleada para mediciones de competitividad donde resaltan diversos estudios, tales como el estudio de Competitividad y empleo en los Sistemas Productivos locales de la CAPV de Larrea (1999), el de Variables e Índices de Competitividad de las Empresas Exportadoras, utilizando el PLS de Bonales, Zamora y Ortiz (2015); Emerging economies' institutional quality and international competitiveness: A PLS-SEM approach de Buitrago R., R. E., Barbosa Camargo, M. I., \& Cala Vitery, F. (2021), entre otros estudios.

No obstante, la relación entre competitividad y desarrollo no se ha abordado a través de la metodología del presente trabajo. Su utilización para la medición de modelos de competitividad obedece a que la técnica PLS presenta varias ventajas en comparación con otras técnicas, al ser más flexible, ya que esta técnica puede utilizar tamaños pequeños de muestra, sin ser necesario que se asuma una distribución normal de los datos, y en las relaciones entre constructos y sus indicadores se pueden incorporar métodos de medida reflectivos y formativos; la PLS tiene como objetivo maximizar la cantidad de varianza explicada (maximiza el coeficiente de determinación $\left[\mathrm{R}^{2}\right]$ ); en la evaluación del modelo global (estimación del modelo de medida) no se establecen criterios de bondad de ajuste, sino que se evalúan por separado las medidas reflectivas y formativas; y el algoritmo básico de la PLS sigue un enfoque de dos pasos, el primero se refiere a la estimación iterativa de las puntuaciones de las variables latentes, y el paso segundo se refiere a la estimación final de los pesos, cargas y coeficientes path por medio de la estimación de mínimos cuadrados ordinarios (múltiples y sencillos), y del análisis de componentes principales (Hair, et al., 2017) y (Henseler, Ringle y Sarstedt, 2015).

\subsection{Variables latentes y observadas}

Las variables denominadas latentes tienen como característica no ser directamente observables o medidas por un instrumento generalmente aceptado (Schumacker y Lomax, 2004). Las variables que contribuyen a formar estas variables latentes son llamadas variables manifiestas, variables observadas o indicadores. En el contexto de Modelación Path PLS, las variables latentes serán obtenidas como una combinación lineal de su grupo de variables observadas (indicadores) (Loehlin, 1988). Se asume que cualquier medición será imperfecta (Bollen, 1989), como lo muestran Haenlein y Kaplan (2004), cada observación en el mundo real viene con un cierto error de medición, el cual puede estar compuesto de dos partes: (a) error aleatorio 
(causado por el orden de los ítems en un cuestionario o sesgo del encuestado), y (b) error sistemático, debido a la varianza del método. Por ello, el valor observado de un ítem es siempre la suma de tres partes, llamadas, el verdadero valor de la variable, el error aleatorio y el error sistemático. Las variables latentes son medidas a través de indicadores, usualmente preguntas en un cuestionario, que son llamadas variables observadas o manifiestas. Por su parte, los errores de medición de los indicadores muestran la porción de este que mide algo distinto a la variable latente (Blunch, 2008).

\section{Variables Seleccionadas}

Con el propósito de comprobar la hipótesis previamente planteada, mediante el uso de la técnica PLS y tomando en consideración a las circunspecciones estipuladas en el párrafo anterior, se seleccionaron para el análisis variables pertenecientes a la dimensión de competitividad internacional y a la dimensión de desarrollo humano de una serie de once países, que forman parte de la región Asia-Pacífico, los cuales son Australia, Canadá, Chile, China, Corea del Sur, Estados Unidos, Japón, México, Nueva Zelanda, Rusia y Singapur. El periodo de tiempo analizado comprende una duración total de siete años, específicamente el periodo del 2010 al 2019. Quedando así, la operacionalización de las variables de la siguiente manera:

a) Dimensión de Competitividad Internacional. La competitividad internacional de estos países se medirá a través de tres variables, cada una de las cuales está compuesta por indicadores que reflejan el comportamiento de la variable. El conjunto total de estos datos se obtuvo de la OECD.

a. Variable de tecnología. Esta se medirá considerando el porcentaje de hogares que en el país cuentan con acceso a Internet mediante banda ancha o ADSL y el número de hogares con al menos una computadora de uso personal en condiciones funcionales, expresada como porcentaje del número total de hogares en el país.

b. Variable Tamaño de mercado. Se calculará a partir del número de personas cuya principal actividad laboral es el autoempleo dentro del país, junto con la cantidad de empresas en el sector manufacturero (corporaciones, cuasicorporaciones, instituciones sin fines de lucro o empresas no incorporadas) que se encuentran operando en el país.

c. Variable comercio. Esta variable se va a definir mediante las exportaciones, importaciones, el valor doméstico cuya acepción es el valor agregado por una economía, al producir bienes y servicios generados para exportación y 
transacciones de bienes y servicios entre residentes y no residentes.

b) Dimensión de Desarrollo Humano. Esta dimensión se basó en el índice de desarrollo humano (IDH) propuesto por el PENUD de la Organización de las Naciones Unidas, dónde a partir de dicho índice, se realiza una medición del nivel de desarrollo humano dentro de los países seleccionados, considerando las siguientes variables:

a. Variable Ingresos. Esta se construye por el PIB per cápita, la tasa de inflación dentro del país y la cantidad de crédito doméstico provisto por el sector financiero como porcentaje del PIB.

b. Variable Educación. Conformada a partir de la cantidad de personas como porcentaje total de la población que han iniciado los estudios en el nivel secundaria, así como la cantidad de años totales de estudio promedio en cada país.

c. Variable Salud. Esta se medirá a partir de la tasa de mortalidad infantil que se presenta en el país, su esperanza de vida y la cantidad de dinero que cada país invierte en el sector salud, expresado como porcentaje del PIB.

Una vez identificadas las dimensiones a analizar y determinar las variables a medir se procede a detallar a continuación los indicadores que se utilizarán para llevar a cabo el modelo PLS, así mismo, se especifican las claves a utilizar dentro del modelo a fin de un mejor reconocimiento y fácil interpretación de los indicadores (véase tabla 1).

\section{Resultados}

El primer análisis que se realiza es el de los efectos, que al permitir evaluar cómo un constructo exógeno contribuye a un constructo latente endógeno, como una medida de relevancia predictiva; el cual puede mostrar un efecto pequeño, medio (alrededor de 0.15$)$ o grande ( 0.35 hacia arriba). De tal manera que se muestran los factores que afectan a cada índice.

Así pues, se puede apreciar que los efectos más grandes corresponden a la relación entre comercio internacional y salud con una carga de 3.029, seguida en orden descendente de la relación de tecnología y salud con 1.008, comercio internacional e ingresos con 0.99, tamaño de mercado y salud con 0.970 , tamaño de mercado e ingresos con 0.956 , tamaño de mercado y educación con 0.92 , comercio internacional y educación 0.923 . 
Tabla 1

Dimensiones, variables e indicadores

\begin{tabular}{|c|c|c|c|}
\hline Dimensión & Variable & Clave & Indicador \\
\hline \multirow{3}{*}{$\begin{array}{l}\text { Dimensión de } \\
\text { Desarrollo } \\
\text { Humano }\end{array}$} & Educación & EDU_AñosEst & $\begin{array}{l}\text { Porcentaje de la población que ha } \\
\text { alcanzado (más no necesariamente } \\
\text { completado) la educación secundaria al } \\
\text { cumplir los } 25 \text { años de edad. } \\
\text { Cantidad de años promedio dedicados a } \\
\text { la educación por ciudadano al alcanzar } \\
25 \text { años de edad. }\end{array}$ \\
\hline & Salud & $\begin{array}{l}\text { SAL_EspVida } \\
\text { SAL_Gast } \% \text { PI } \\
\text { B } \\
\text { SAL_MortInf }\end{array}$ & $\begin{array}{l}\text { Esperanza de vida. } \\
\text { Gasto porcentual del PIB en el sector } \\
\text { salud } \\
\text { Tasa de mortalidad infantil (por cada } \\
1000 \text { nacimientos con vida). }\end{array}$ \\
\hline & Ingresos & $\begin{array}{l}\text { ING_PIBpC } \\
\text { ING_Cred } \\
\text { ING_Inf }\end{array}$ & $\begin{array}{l}\text { PIB per cápita. } \\
\text { Crédito doméstico dado por el sector } \\
\text { financiero (expresado como porcentaje } \\
\text { del PIB). } \\
\text { Inflación medida por índice de precios al } \\
\text { consumidor. }\end{array}$ \\
\hline \multirow{3}{*}{$\begin{array}{l}\text { Dimensión de } \\
\text { Competitividad } \\
\text { Internacional }\end{array}$} & $\begin{array}{l}\text { Tamaño de } \\
\text { mercado }\end{array}$ & $\begin{array}{l}\text { MER_AutoE } \\
\text { MER_Emp }\end{array}$ & $\begin{array}{l}\text { Porcentaje de la población } \\
\text { económicamente activa que se encuentra } \\
\text { en el auto-empleo. } \\
\text { Número de empresas registradas en el } \\
\text { sector manufacturero. }\end{array}$ \\
\hline & Tecnología & TEC_ComCas & $\begin{array}{l}\text { Porcentaje de hogares con acceso a } \\
\text { internet (mediante un dial-up, ADSL o } \\
\text { cable de banda ancha) } \\
\text { Porcentaje de hogares que cuentan con al } \\
\text { menos una computadora personal } \\
\text { funcional. }\end{array}$ \\
\hline & $\begin{array}{l}\text { Comercio } \\
\text { Internacional }\end{array}$ & $\begin{array}{l}\text { COM_Exp } \\
\text { COM_Imp }\end{array}$ & $\begin{array}{l}\text { Exportaciones. } \\
\text { Importaciones. } \\
\text { Transacciones de bienes y servicios } \\
\text { entre residentes y no-residentes, medido } \\
\text { en millones de USD como porcentaje del } \\
\text { PIB por comercio neto*. } \\
\text { La diferencia entre la producción bruta a } \\
\text { precios básicos y el consumo intermedio } \\
\text { en precio de comprador, expresado como } \\
\text { porcentaje del valor final. }\end{array}$ \\
\hline
\end{tabular}

Fuente: Elaboración propia.

Tabla 2

Efectos totales

\begin{tabular}{llll}
\hline & Educación & Ingresos & Salud \\
\hline Tamaño de mercado & 0.925 & 0.956 & 0.970 \\
Tecnología & 0.335 & 0.814 & 1.008 \\
Comercio Internacional & 0.923 & 0.999 & 3.029 \\
\hline
\end{tabular}

Fuente: Elaboración propia con base en el modelo PLS.

Cabe recordar que el objetivo primario del modelo de $P L S$ es la predicción, y es pertinente evaluar la bondad del modelo mediante dos índices principales: 
los coeficientes de los paths estructurales y la predictividad combinada (R2) de los constructos endógenos (Chin, 2010).

Acorde con el criterio utilizado por Duarte y Raposo (2010), la varianza explicada (R2) para las variables endógenas debe ser mayor que 0.1. En la tabla 3, se observa el comportamiento de la varianza explicada o las $\mathrm{r}$ cuadradas, donde todas las variables muestran valores por arriba del 0.1. Así mismo se observa que la variable ingresos presenta los indicadores más altos, el R2 por 0.9240 y el R2aj 0.8714 , seguido de la variable salud y la variable educación, como también se pueden ver de manera gráfica en la figura 1.

Tabla 3

R Cuadradas

\begin{tabular}{lll}
\hline & R Square & R Square Adjusted \\
\hline Educación & 4.577 & 3.945 \\
Ingresos & 9.240 & 8.714 \\
Salud & 9.348 & 8.720 \\
\hline
\end{tabular}

Fuente: Elaboración propia con base en el modelo PLS.

El proceso que sigue la modelación con el PLS inicia con la evaluación de los efectos directos o también conocidos como valores de paths, midiendo los pesos de las relaciones entre indicadores y sus variables respectivas, posteriormente, se realiza el cálculo de los case values para cada variable latente, para ello, se toma el promedio ponderado de sus indicadores, se evalúan los efectos totales entre las variables, que son la suma de los efectos directos e indirectos, a través de un diagrama de trayectoria o path diagram de tal forma que se muestran así las variables que afectan mayormente a cada índice de competitividad.

Los case values son valorados en un grupo de ecuaciones de regresión para determinar los parámetros de los coeficientes estructurales o paths (Haenlein y Kaplan, 2004). Como se observa en la figura 1, el algoritmo regresa nuevamente a las relaciones del modelo de medición en donde nuevos pesos (outer weights) son calculados, y el proceso continúa iterativamente hasta que se alcanza la convergencia de los pesos.

En cuanto al peso de las relaciones causales, se observa que la relación más débil se obtiene de la relación de la variable comercio internacional respecto de la variable salud, donde apenas se percibe un impacto. Mientras que los pesos más significativos, se obtienen de la relación entre las variables tecnología y educación con un valor de 0.734 , seguido de tecnología e ingreso con un valor de 0.418 , lo cual pone de manifiesto la importancia del desarrollo tecnológico e innovación como un mecanismo para generar condiciones para 
el desarrollo humano, a través de la capacitación de las nuevas generaciones (ver tabla 4).

Tabla 4

Relaciones entre variables

\begin{tabular}{llll}
\hline Relación entre variables & Coeficientes & T-Statistics & P-Values \\
\hline Comercio Internacional -> Educación & 0.356 & 0.923 & 0.356 \\
Comercio Internacional -> Ingreso & 0.317 & 0.999 & 0.318 \\
Comercio Internacional -> Salud & 0.004 & 3.029 & 0.002 \\
Tamaño de mercado-> Educación & 0.353 & 0.925 & 0.355 \\
Tamaño de mercado-> Ingreso & 0.339 & 0.956 & 0.339 \\
Tamaño de mercado-> Salud & 0.334 & 0.970 & 0.332 \\
Tecnología -> Educación & 0.734 & 0.335 & 0.738 \\
Tecnología -> Ingreso & 0.418 & 0.814 & 0.415 \\
Tecnología -> Salud & 0.269 & 1.008 & 0.314 \\
\hline
\end{tabular}

Fuente: Elaboración propia con base en el modelo PLS.

Dando como resultado un modelo estructural en forma gráfica que representa las relaciones entre constructos que son hipotetizadas en el modelo propuesto. Para analizar el modelo estructural con PLS, este debe ser planteado como un modelo recursivo, lo que significa que no se permiten loops en las relaciones estructurales. Después de procesar la técnica del Bootstrapping con 2000 observaciones con un margen de error de 0.5 , se presentan los siguientes resultados para el caso del presente estudio, donde la variable con mayor peso respecto de la dimensión de competitividad (considerando las variables tecnología, comercio internacional y tamaño de mercado), es educación con un factor de 0.936 , seguido por salud con 0.520 e ingreso con 0.477 , dando un promedio total de la dimensión de desarrollo humano 0.6443 .

Así mismo, resulta interesante analizar qué variables cobran más importancia para la conjugación de variables, por ejemplo, se pude apreciar que para el clúster salud, la esperanza de vida es la más significativa, seguida del gasto en salud como porcentaje del PIB y, por último, la mortalidad infantil, aunque con una ponderación similar. En el caso de la variable ingresos, el peso es para el PIB per cápita, seguido del crédito y la inflación. Mientras que, en el caso de la educación, los años de estudio tienen una carga más alta que la población con educación secundaria. 
Figura 1

Interrelación de las variables de competitividad y desarrollo humano

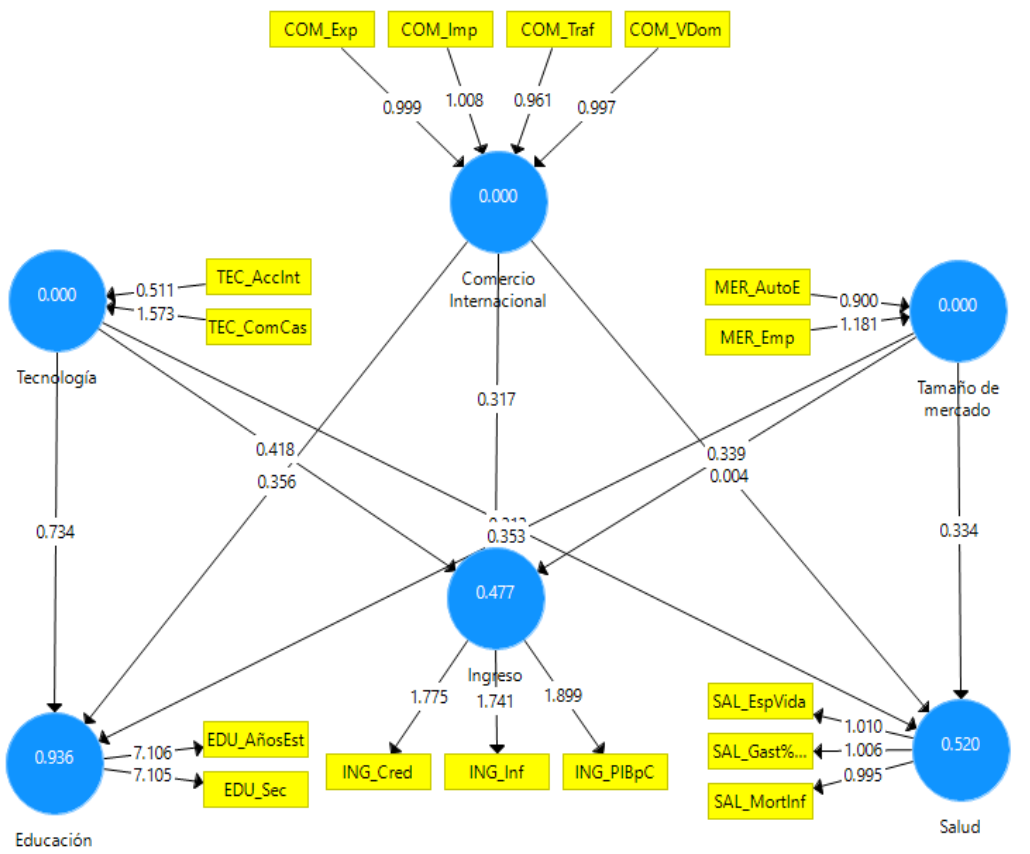

Fuente: Elaboración propia con base en el modelo PLS.

Al observar las tres variables analizadas (educación, ingresos y salud), es educación la variable donde los indicadores de competitividad tienen más impacto, con un valor de 0.936 .

Como se puede apreciar se dio respuesta al objetivo de la presente investigación, al identificar las interrelaciones entre las variables de competitividad internacional (tecnología, comercio internacional y tamaño de mercado) respecto de las variables de desarrollo humano, como son educación, ingreso y salud para la Región Asia Pacifico. Observando que existe un impacto positivo de las primeras variables sobre las segundas, pese a que este impacto no fue tan grande como se esperaría, siendo el valor más alto de las relaciones causadas de 0.734 de la variable tecnología a la variable educación. Que, sin duda, ahora después de las adecuaciones al sistema educativo, con motivo de la pandemia Covid-19, tendrá un peso aún más significativo. 
De acuerdo con el objetivo planteado, si el desarrollo humano y la competitividad están correlacionadas, ¿qué variables son las más importante para el bienestar económico de un país, una región, una industria y una empresa? De manera más específica, acorde con el modelo presentado y sus respectivas dimensiones, variables e indicadores, el impacto más grande de la competitividad en el desarrollo humano se da a través de la variable ingresos. El análisis continuo de la literatura confirma esta relación directa entre estos dos constructos. Sin sectores, industrias y/o empresas competitivas, el nivel de vida, la creación de bienestar económico, el nivel de ingresos, el nivel de empleo, la calidad de vida, la prosperidad económica, la distribución del ingreso, la riqueza, el pleno empleo de una nación será menor, ya que tendrá que renunciar a más producción para pagar sus importaciones. Así mismo, se observa una interrelación en salud y educación respecto de la competitividad, siendo esta menos pronunciada en el modelo expuesto, lo que pone de manifiesta la necesidad de que los gobiernos articulen estrategias para que la productividad permee a los diferentes sectores de la sociedad generando un bienestar real en las sociedades.

Cabe señalar que el presente modelo tiene como limitantes los propios indicadores utilizados, así como el set de países escogidos para su realización, por lo que se proponen para futuras líneas de investigación realizar un estudio similar, pero con más países o diferentes regiones, a fin de observar si se replican estos resultados.

\section{Discusión}

Se ha llevado a cabo en los últimos años un intenso debate académico, económico, político y social sobre cómo mejorar las formas de conceptualizar y medir la competitividad. La teoría clásica de la ventaja comparativa ha regulado durante mucho tiempo el pensamiento sobre el comercio internacional; actualmente, se reconoce como una explicación incompleta de la ventaja competitiva en todos los niveles (país, región, industria y empresa). Las innovaciones y los avances tecnológicos, los problemas ecológicos, y la escasez de recursos han creado nuevas oportunidades y limitaciones para ganar, mantener y mejorar la competitividad frente a los rivales en una economía cada vez más compleja y globalizada, considerando como objetivo de la competitividad la prosperidad de las naciones.

Como lo señala la revisión de literatura, muchos autores señalan que el bienestar nacional es determinado por la competitividad, la cual a su vez se instrumenta a través de la productividad con la que la nación utiliza sus insumos (recursos). No obstante, resulta importante analizar qué variables tienen un impacto real en el crecimiento y desarrollo de las sociedades, a fin 
de focalizar esfuerzos entorno a medidas que impacten verdaderamente en el bienestar de las personas.

Al referirse a la competitividad de las economías mundiales, se puede complicar la tarea de esclarecer cómo las variables que tienen impacto en esta, causan dicho impacto o bien, cómo causan efecto en el desarrollo humano de la población y mientras algunos autores encuentran una relación estrecha, otros difieren de que incluso exista un impacto real de estas variables en los indicadores básicos para determinar desarrollo humano.

Considerando los resultados y el impacto que tiene la variable tecnología en la educación, aunado a los cambios que este sector se ha visto forzado a implementar debido al Covid -19, no solo es importante sino además necesaria la incorporación de las TIC en la educación formulando políticas públicas, presentando su incidencia en el aprendizaje, su rol frente a la sociedad de la información, los desafíos de la educación inclusiva, así como de las tecnologías emergentes. Así pues, las políticas públicas deberán enfocarse en la inversión en ciencia y tecnología, capacitación del profesorado en la incorporación de las TIC, como herramientas permanentes y no emergentes del sistema educativo.

Así mismo, considerando que la tecnología también genera un impacto positivo en la variable ingreso, se propone generar políticas públicas en torno al fomento de la innovación y desarrollo tecnológico, a fin de promover una economía competitiva que impacte verdaderamente en el desarrollo de la sociedad.

Por su parte, partiendo de que la variable Tamaño de mercado, medida a través del número de empresas y el auto empleo, mostró impactar en los tres factores de desarrollo (ingresos, educación y salud), es una variable a cuidar a través de políticas que incentiven, apoyen y promuevan la generación de empresas en las sociedades, así como en épocas de crisis como la que actualmente se vive, protejan a la industria y, por ende, los beneficios que se desprenden de ellas.

\section{Conclusiones}

El presente artículo demuestra cómo la Modelación de Ecuaciones Estructurales mediante la técnica de Partial Least Squares (PLS-SEM), así como mediante el muestreo y remuestreo llevado a cabo por el Bootstrapping de 2000 observaciones, puede ser aplicada exitosamente en modelos complejos que buscan explicar la realidad de sistemas macroeconómicos comprendidos entre una variedad amplia de elementos diversos y su impacto en las variables de desarrollo humano, junto con sus respectivas interrelaciones. 
Para el caso del presente estudio, desarrollado a través de la técnica de PLS, partiendo de las variables consideradas para el caso de los países de la región Asia-Pacífico, es posible observar una relación entorno a las variables tecnología, comercio internacional y tamaño de mercado (variables típicamente usadas para medir la competitividad de las economías), respecto del nivel de desarrollo humano medido a través de las variables de educación, ingresos y salud.

\section{Referencias}

[1] Abdel, G. y Romo, D. (2004). Sobre el concepto de competitividad en coord. Santiago Macias y Víctor Mirlos, Capacitación y competitividad. México, Grupo Noriega Editores, 288.

[2] APEC (2021). Asia Pacific Economic Cooperation. StatsAPEC http://statistics.apec.org/index.php/apec_psu/index

[3] Ávila, L. (2015). Integración de México con Asia: Acuerdo Estratégico Transpacífico de Asociación Económica (TPP). En L. A. Jiménez, Integración de México a las dinámicas globales y la potencialidad de la Alianza del Pacífico, Tijuana: Red de Relaciones Internacionales y Desarrollo Global, 291-298.

[4] Barro, R., \& Sala-i-Martin, X. (2012). Crecimiento Económico. Barcelona: Revertè.

[5] Batista Foguet \& Coenders Gallart, (2000). Modelos de ecuaciones estructurales. Ed. Hespérides, España

[6] Blunch, N. J. (2008). Introduction to structural equation modelling using SPSS and AMOS. SAGE.

[7] Bonales, J.; Zamora, A.; y Ortiz, C. (2015). "Variables e índices de Competitividad de las Empresas Exportadoras, utilizando el PLS". Cimexus, ISSN 18706479, ISSN-e 2007-9206, Vol. 10, No. 2, (Julio - Diciembre), 13-32.

[8] Boone, J. (2008). "New Way to Measure Competition". Economics Journals. 2008; 118 (531):1245-61. DOI: 10.1111/j.1468-0297.2008.02168.x

[9] Bosse, D., Philips, R. y Harrison, J. (2009). "Stakeholders, reciprocity and firm performance". Strateic Management Journal, 30(4), 447-456.

[10] Buckley, P., Pass C. y Prescott K. (1988). "Measures of international competitiveness: A critical survey". J Mark Management 4(2):175-200

[11] Buendía, E. (2013). "El papel de la Ventaja Competitiva en el desarrollo económico de los países". Análisis Económico. Núm. 69, vol. XXVIII Tercer cuatrimestre, 55-78.

[12] Buitrago, R., R. E., Barbosa Camargo, M. I., \& Cala Vitery, F. (2021). "Emerging economies' institutional quality and international competitiveness: A PLSSEM approach". Mathematics. https://www.mdpi.com/22277390/9/9/928/htm

[13] Calva, J. L. (2009). Moritz, Situación y perspectiva de la economía mexicana, invierno 2008-2009, Instituto de Investigaciones Económicas, UNAM, México.

[14] Cepeda, G, y Roldán, J. (2004). "Aplicando en la práctica la técnica PLS en la administración de empresas". Revista de la Universidad de Sevilla, España. Recuperado 
https://personal.us.es/jlroldan/Sitio_web/Partial_Least_Squares_(PLS)_files /Cepeda,\%20Roldan\%20\%282004\%29\%20ACEDE.pdf

[15] Chin, W. W. (2010). "How to Write Up and Report PLS Analyses". En V. Esposito Vinzi, W. W. Chin, J. Henseler, y H. Wang (Eds.), Handbook of Partial Least Squares: Concepts, Methods and Applications, 1, 655-690. Berlin, Heidelberg: $\quad$ Springer Berlin Heidelberg. https://link.springer.com/chapter/10.1007\%2F978-3-540-32827-8_29

[16] Chin, Wynne \& Marcoulides, G.. (1998). "The Partial Least Squares Approach to Structural Equation Modeling”. Modern Methods for Business Research. 8.

[17] Constanza, R., Fisher, B., Ali, S., Beer, C., Bond, L., Boumans., Danigelis, N. L., Dickinson, J., Elliot, C., Farley, J., Gayer, D. E., Glenn, L. M., Mahoney, D. F., McCahill, L., McIntosh, B., Reed, B., Rizvi, A. T., Rizzo, D. M., Simpatico, T. \& Snapp, R. (2007). "An Integrative Approach to Quality of Life Measurement, Research, and Policy". Surveys and Perspectives Integrating Environment and Society. 1(1) 1-6.

[18] Cruz, Moritz. (2007). "La globalización como estrategia de desarrollo: la evidencia de los países desarrollados”. Investigación económica, 66(259), 103-131. Recuperado en 08 de junio de 2021, de http://www.scielo.org.mx/scielo.php?script=sci_arttext\&pid=S0185$16672007000100103 \& \operatorname{lng}=$ es\&tlng=es

[19] Dixit, A. y Stiglitz, J. (1977). "Monopolistic Competition and Optimum Product Diversity". The American Economic Review, 67(3), 297-308. http://www.jstor.org/stable/1831401.

[20] Fornell, C. (1982). A Second Generation of Multivariate Analysis: An Overview. en C. Fornell. Ed. A Second Generation of Multivariate Analysis.

[21] Garduño, R., Ibarra, J. E. \& Dávila, R. (2013). "La Medición de la Competitividad en México: Ventajas y Desventajas de los Índicadores". Realidad, Datos y Espacio. Revista Internacional de Estadística y Geografía. 4(3), 28-53.

[22] Haenlein, M., and Kaplan, A. (2004). A beginner's guide to partial least squares analysis. Understanding Statistics, 3 (4), 283-97.

[23] Hair, J., Hult, G., Ringle, C. y Sarstedt, M. (2017). A Primer on Partial Least Square Structural Equation Modeling (PLS-SEM). Estados Unidos, California: Sage.

[24] Helliwell, J. F., Layard, R., Sachs J \& De Neve J-E. (2020). World Hapiness Report. Primera edición. Nueva York, USA: Sustainable Development Solutions Network.

[25] Henseler, J., Ringle, C. y Sarstedt, M. (2015). "A new criterion for assessing discriminant validity in variance-based structural equation modeling". Journal of the Academy of Marketing Science, 43(1), 115-135.

[26] INEGI (Instituto Nacional de Estadística, Geografía e Informática) (1998). "Indicadores de competitividad de la economía mexicana". Aguascalientes, México.

[27] Jiménez, F. (2011). Crecimiento Económico Enfoques y Modelos. Lima, Perú: Fondo Editorial.

[28] Johnson, R. (1997). Examining the Validity Structure of Qualitative Research. Educ. 118.

[29] Krugman, P. (1979a). "A model of innovation, technology transfer, and the world distribution of income". Journal of Political Economy, 87 (2), 253-266. doi: $10.1086 / 260755$ 
[30] Krugman, P. (1979b). "Increasing returns, monopolistic competition and international trade". Journal of International Economics, 9(4), 469-479. doi:10.1016/0022-1996(79)90017-5

[31] Krugman, P. (1990). "Rethinking international trade". Cambridge, MA: MIT Press.

[32] Krugman, P. (1991). "Increasing returns and economic geography". Journal of Political Economy, 99, 483-499. doi: 10.1086/261763

[33] Krugman, P. (1994). “Competitiveness: A dangerous obsession”. Foreign Affairs, 73(2), 28-44. doi: 10.2307/20045917

[34] Krugman, P. (1996). "Making Sense of the Competitiveness Debate". Oxford Review of Economic Policy, 12 (3), pp. 483- 499.

[35] Larrea, J. L. (2018). "Desafíos a la Competitividad desde el Bienestar y la Cohesión Social". Cuadernos Orkestra, 35. 1-105.

[36] Larrea, M. (1999). "Competitividad y empleo en los Sistemas Productivos locales de la CAPV”. Ekonomiaz: Revista vasca de economía, ISSN 0213-3865, No. 44, 1999, 144-173.

[37] Loehlin, J. (1998). Latent Variable Models. An introduction to factor, path, and structural analysis, Lawrence Eribaum Associates Inc., Publishers, New Jersey.

[38] Lombana, Jahir, \& Rozas Gutiérrez, Silvia. (2009). Marco analítico de la competitividad: Fundamentos para el estudio de la competitividad regional. Pensamiento \& Gestión, (26), 1-38. Retrieved June 08, 2021, from http://www.scielo.org.co/scielo.php?script=sci_arttext\&pid=S1657$62762009000100002 \& \operatorname{lng}=$ en\&tlng=es

[39] Lonska, J., \& Boronenko, V. (2015). Rethinking Competitiveness and Human DevelopmentinGlobal Comparative Researches. Procedia Economics and Finance, 1030 - 1036.

[40] Malecki, E (1997). “Technology and Economic Development”. Longman, Londres.

[41] Marsh, I. y S. Tokarik (1994). "Competitiveness Indicators: a Theoretical and Empirical Assessment”. International Monetary Found, Nueva York, Working Paper 94/29.

[42] Mas, M., Paluzie, E., Pons, J., Quesada, J., Robledo, J. C. \& Tirado, D. A. (2007). Competitividad, Crecimiento y Capitalización de las Regiones Españolas. Primera edición. Bilbao, España: Fundación BBVA

[43] Miquel, A. (2015a). El impacto del crecimiento sobre el bienestar económico sostenible de las naciones: análisis crítico. Departamento de Economía Aplicada e Historia Económica Facultad de Ciencias Económicas y Empresariales. UNED.

[44] Miquel, A. (2015b). "La Medición del Bienestar Económico a través de las Macromagnitudes de la Contabilidad Nacional Ciriec-España", Revista de Economía Pública, Social y Cooperativa. № 85/2015, 245-286.

[45] Morris, D. (1979) "Measuring the condition of the world's poor: The Physical Quality of Life Index". Pergamon for the Overseas Development Council. 42. 1-176.

[46] Mussi, C y Afonso, J. R. (2008). “CCómo conciliar desarrollo económico con bienestar social? Algunas reflexiones sobre los nuevos desafíos latinoamericanos". Nueva Sociedad, 215. mayo-junio de 2008, ISSN: 02513552.

[47] Noyola, P. (1991), “La competitividad de la economía mexicana”. en L. Mercado (comp.), Competitividad, segundo seminario de El Economista, pp. 21-32. 
[48] OCDE (2015). Estudios Económicos de la OCDE Chile. OCDE.

[49] Ordóñez, J. A. (2011). “CCompetitividad para qué? Análisis de la relación entre competitividad y desarrollo humano en México". Revista del Centro Latinoamericano de Administración para el Desarrollo Reforma y Democracia. 51. 2-20.

[50] Ortega, O. y Bonales, J. (2014). "Modelación para la interreación entre factores de competitividad de las empresas agroindustriales del Estado de Michoacán”. Revista Nicolaita de Estudios Económicos, Vol IX, No. 2. Julio-diciembre, 141-172.

[51] Porter, M. (1990). “The Competitive Advantage of Nations”. EE. UU.: New York, Free Press, New York.

[52] Porter, M. (1991). “Toward a Dynamic Theory of Strategy”. Strategic Management Journal, 12, 95-117.

[53] Prahalad, C. y Hamel, G. (1990). "The Core Competence of the Corporation". Harvard Business Review, 68, 79-90.

[54] Programa de las Naciones Unidas para el Desarrollo (2019). Informe sobre Desarrollo Humano 2019. Primera edición. Nueva York, USA: Programa de las Naciones Unidas para el Desarrollo (PNUD)

[55] Reyes, J. (2010). “El residuo de Solow”. [versión electrónica]. Revista de Economía Institucional, 12, 23, segundo semestre de 2010.

[56] Schumacker, R. y Lomax, R. (2004). A beginner's guide to structural equation modeling. 2nd edition. Lawrence Eribaum Associates, Inc

[57] Siudek, T. y Zawojska, A. (2014). "Competitiveness in the economic concepts, theories and empirical Research". Acta Science Polonorum. 2014;13(1):91108.

[58] Wysokinska, Z. (2003).“Competitiveness and its relationships with productivity and sustainable development”. Fibres Text East Europe. 11(3):11-4. 